\title{
Inhibition of the HDAC/Suv39/G9a pathway restores the expression of DNA damage-dependent major histocompatibility complex class I-related chain $A$ and $B$ in cancer cells
}

\author{
NAKAKO IZUMI NAKAJIMA ${ }^{1}$, ATSUKO NIIMI ${ }^{2}$, MAYU ISONO ${ }^{3}$, TAKAHIRO OIKE ${ }^{4}$, \\ HIRO SATO $^{4}$, TAKASHI NAKANO ${ }^{4}$ and ATSUSHI SHIBATA ${ }^{3}$
}

\begin{abstract}
${ }^{1}$ Radiological Science Research and Development Directorate, National Institutes for Quantum and Radiological Science and Technology, Chiba 263-8555; ${ }^{2}$ Research Program for Heavy Ion Therapy, Division of Integrated Oncology Research, Gunma University Initiative for Advanced Research; ${ }^{3}$ Advanced Scientific Research Leaders Development Unit and ${ }^{4}$ Department of Radiation Oncology, Gunma University, Maebashi, Gunma 371-8511, Japan
\end{abstract}

Received October 3, 2016; Accepted May 5, 2017

DOI: $10.3892 /$ or.2017.5773

\begin{abstract}
Immunotherapy is expected to be promising as a next generation cancer therapy. Immunoreceptors are often activated constitutively in cancer cells, however, such levels of ligand expression are not effectively recognized by the native immune system due to tumor microenvironmental adaptation. Studies have demonstrated that natural-killer group 2, member D (NKG2D), a major activating immunoreceptor, responds to DNA damage. The upregulation of major histocompatibility complex class I-related chain A and B (MICA/B) (members of NKG2D ligands) expression after DNA damage is associated with NK cell-mediated killing of cancer cells. However, the regulation of DNA damage-induced MICA/B expression has not been fully elucidated in the context of the types of cancer cell lines. In the present study, we found that MICA/B expression varied between cancer cell lines after DNA damage. Screening in terms of chromatin remodeling identified that inhibitors related to chromatin relaxation via post-translational modification on histone H3K9, i.e. HDAC, Suv39 or G9a inhibition, restored DNA damage-dependent MICA/B expression in insensitive cells. In addition, we revealed that the restored MICA/B expression was dependent on ATR as well as E2F1, a transcription factor. We further revealed that low-dose treatment of an HDAC inhibitor was sufficient to restore MICA/B expression in insensitive cells. Finally, we demonstrated that HDAC inhibition restored DNA damage-dependent cytotoxic NK activity against insensitive cells. Thus, the present study revealed that DNA damage-dependent MICA/B expression in
\end{abstract}

Correspondence to: Dr Atsushi Shibata, Advanced Scientific Research Leaders Development Unit, Gunma University, 3-39-22 Showa-machi, Maebashi, Gunma 371-8511, Japan

E-mail: shibata.at@gunma-u.ac.jp

Key words: immunotherapy, NKG2D ligand, HDAC inhibitor, chromatin remodeling, ionizing radiation insensitive cancer cells can be restored by chromatin relaxation via the HDAC/Suv39/G9a pathway. Collectively, manipulation of chromatin status by therapeutic cancer drugs may potentiate the antitumor effect by enhancing immune activation following radiotherapy and DNA damage-associated chemotherapy.

\section{Introduction}

Immune suppression in cancer patients is one of major issues that promotes tumor progression and inhibits the efficiency of anticancer treatment. DNA damage response in cancer cells following radiotherapy or chemotherapy engages host immune effectors that may contribute to eradication of cancer. Nevertheless, in many cases, it is likely that the stimulation in response to DNA damage is insufficient to activate the immune system in order to overcome the cancer. Therefore, enhancement of immune activity with additional therapeutic drugs may be promising to increase the antitumor effect in combination with radiotherapy or chemotherapy.

To improve the efficacy of immunotherapy, numerous studies have sought to develop novel strategies including immune-checkpoint inhibitors that can overcome the immunosuppressive environment (1-3). Among the several immune systems, one of the major activating immunoreceptors, natural-killer group 2 member D (NKG2D), plays a central role in the antitumor effect. NKG2D is expressed in several types of immune cells, including NK, NKT, $\gamma \delta \mathrm{T}$ and $\mathrm{CD}^{+} \alpha \beta \mathrm{T}$ cells that exert cytotoxicity against cancer cells $(4,5)$. Therefore, reactivation of the NKG2D-mediated immune response is important for invoking an initial response in terms of cancer immunotherapy. Major histocompatibility complex class I-related chains A and B (MICA/B), ligands for the NKG2D immune receptor, in human cells are often constitutively upregulated in cancer cells, but are not effectively recognized in an immunosuppressive environment (6). Regardless of the adapted environment, further activation of MICA/B is able to promote an antitumor effect. For example, DNA damage is one of the activators of MICA/B in cancer cells. Consistent with the evidence of the upregulation of 
MICA/B expression by DNA damage, ionizing radiation (IR) and chemotherapeutic agents enhance NK cell-mediated killing of cancer cells (7-9). DNA double-strand breaks (DSBs) are the most critical DNA lesions induced by IR. Gene expression following DNA damage is regulated by kinases that are also involved in repair and cell-cycle checkpoint arrest (10). Ataxia telangiectasia, mutated (ATM), which activates the repair and checkpoint machinery, is a 'first responder' to DSBs (11). During homologous recombination (HR), a major DSB repair pathway, cells activate ATM- and Rad3-related (ATR) protein kinase, followed by Chk1 $(11,12)$. Previous studies have revealed that the ATR/Chk1 pathway plays an important role in the expression of MICA/B after DNA damage. However, although the requirement of DNA damage signaling in MICA/B expression has been reported, variations in the responsiveness of MICA/B among cancer cell lines after DNA damage have not been thoroughly investigated (7).

In the present study, we first investigated DNA damagedependent MICA/B expression on the surface of cancer cells in relation to tissue type and tumor-suppressor gene status. Neither tissue type nor Rb/p53 status influenced MICA/B expression. Notably, however, various cancer cell lines did not express MICA/B in response to IR or aphidicolin, a DNA polymerase inhibitor which induces DNA damage by blocking DNA replication. These insensitive cell lines did not respond even after carbon-ion irradiation, which induces robust DNA damage signaling. Next, we screened compounds that affect chromatin relaxation since we hypothesized that MICA/B gene expression in insensitive cells may be suppressed by chromatin disorganization in the tumor environment. We found that treatment with a histone deacetylase inhibitor (HDACi; vorinostat) restored DNA damage-induced MICA/B expression in insensitive cells. In addition, inhibition of Suv39 or G9a histone methyltransferase activity, both of which promote chromatin relaxation via the HDAC axis, restored MICA/B expression in insensitive cells after IR (13-15). The restored MICA/B expression by HDACi was prevented by inhibition of ATR or depletion of E2F1. Furthermore, our titration analysis revealed that a low-dose of HDACi was sufficient to restore DNA damage-dependent MICA/B expression in insensitive cells. Finally, inhibition of HDAC restored IR-dependent cytotoxic NK activity in insensitive cells. Thus, MICA/B expression in insensitive cells can be restored by therapeutic HDACi or inhibition of Suv39/G9a activity. Collectively, our results demonstrate that inhibition of the HDAC/Suv39/G9a pathway may potentiate the therapeutic efficacy of radiotherapy and DNA-damaging chemotherapy by reactivating MICA/B-mediated killing of cancer cells.

\section{Materials and methods}

Cell lines, reagents and irradiation. The cancer cell lines were cultured in Dulbecco's modified Eagle's medium, RPMI-1640 medium (both from Wako, Tokyo, Japan) or McCoy's 5A medium (Gibco, Carlsbad, CA, USA) supplemented with $10 \%$ fetal calf serum (FCS) at $37^{\circ} \mathrm{C}$ in a humidified mixture of $95 \%$ air and $5 \% \mathrm{CO}_{2}$. X-rays were generated at $200 \mathrm{kVp}$ and $20 \mathrm{~mA}$ using copper $(0.5 \mathrm{~mm})$-aluminum $(1.0 \mathrm{~mm})$ filters. Exposure to carbon-ion irradiation $(290 \mathrm{MeV} / \mathrm{n}, \mathrm{LET} \sim 70 \mathrm{keV} / \mu \mathrm{m})$ was performed at the Heavy Ion Medical Accelerator (HIMAC) facility of the National Institute of Quantum and Radiological Science and Technology (Chiba, Japan), or at the Gunma University Heavy Ion Medical Center (GHMC; Gunma, Japan) (290 MeV/n, LET $70 \mathrm{keV} / \mu \mathrm{m})$. Before IR, $10 \mu \mathrm{M}$ of ATR inhibitor (ATRi), VE821 (Axon), was added at $30 \mathrm{~min}$. At this concentration, the drug specifically inhibited the target. The DNA polymerase inhibitor aphidicolin (Wako) was added at a final concentration of $4 \mu \mathrm{M}$, and the cultures were then incubated for $24 \mathrm{~h}$.

Analysis of MICA/B surface expression by fluorescence-activated cell sorting (FACS). Cancer cells were incubated for $24 \mathrm{~h}$ after exposure to 10 Gy X-rays, carbon ions, or aphidicolin treatment, and then harvested for FACS analysis. Adherent cells were harvested by shake-off in $2 \mathrm{mM}$ EDTA/phosphate-buffered saline (PBS) without trypsinization according to the method described by Clayton et al (16). Harvested cells were washed with FACS solution (ice-cold PBS containing $2 \%$ newborn calf serum, $1 \mathrm{mM}$ EDTA, $0.01 \% \mathrm{w} / \mathrm{v} \mathrm{NaN}$ ), and then stained with MICA/B antibodies for $20 \mathrm{~min}$ at $4^{\circ} \mathrm{C}$. Cells undergoing apoptosis were detected using Annexin V. MICA/B expression was analyzed in cells doubly negative for propidium iodide (PI) (Sigma-Aldrich, St. Louis, MO, USA) and Annexin V (BioLegend, San Diego, CA, USA). FACS was performed on a FACSCalibur instrument using the CellQuest software. FACS data were analyzed using the FlowJo v. 9.3 software (Tree Star, Inc., Ashland, OR, USA). Expression levels of surface MICA/B were determined as mean fluorescence intensity (MFI) of anti-MICA/B normalized against the MFI of an isotype control antibody. The IR-induced fold increase in expression level was calculated by dividing the MFI of irradiated cells (IR-MFI) by the MFI of non-irradiated cells (non-IR-MFI). Reproducible results in all FACS experiments were obtained from two or more independent experiments. A representative FACS histogram is shown for each analysis.

Drug screening focusing on factors that influence chromatin remodeling. The $\mathrm{T} 98 \mathrm{G}$ cell line, an insensitive cell line, was used in the screening analysis. Each inhibitor was added $2 \mathrm{~h}$ before cells were exposed to X-rays, and the cells were harvested $24 \mathrm{~h}$ post-IR. MICA/B expression was analyzed by FACS. Drug information, including concentration in the media, is listed in Table I.

siRNA transfection. siControl and siE2F1 were obtained from Ambion Silencer ${ }^{\circledR}$ Select siRNA (Life Technologies, Carlsbad, CA, USA). siRNA transfection was performed using HiPerFect (Qiagen, Valencia, CA, USA) as previously described (17). Briefly, siRNA transfection was carried out in suspended cells following trypsinization. After $24 \mathrm{~h}$, cells were re-transfected with siRNA in suspension following trypsinization. Prior to analysis, cells were incubated for $48 \mathrm{~h}$ after the second transfection.

Immunofluorescence staining and microscopy. Immunofluorescence (IF) staining was performed as previously described (18). Images were captured on an Olympus BX51 microscope with identical exposure times. 
Table I. Inhibitors used in the present study.

\begin{tabular}{llllr}
\hline Name & \multicolumn{1}{c}{ Effect } & \multicolumn{1}{c}{ Supplier } & Cat. no. & Dose \\
\hline Vorinostat (SAHA) & HDAC inhibitor & Focus Biomolecules & $10-1067$ & $1 \mu \mathrm{M}$ \\
TSA & HDAC inhibitor & Wako Pure Chemical Industries & $203-17561$ & $100 \mathrm{nM}$ \\
Chaetocin & Suv39 histone methyltransferase inhibitor & Abcam & ab144534 & $50 \mathrm{nM}$ \\
Bix-01294 & G9a histone methyltransferease inhibitor & Cayman Chemical Company & 13124 & $5 \mu \mathrm{M}$ \\
5-Aza-cytidine & DNA methyltransferase inhibitor & TCI Chemicals & A2033 & $1 \mu \mathrm{M}$ \\
Zebularine & DNA methyltransferase inhibitor & Tocris Bioscience & 2293 & $10 \mu \mathrm{M}$ \\
NU9056 & HAT inhibitor KAT5, p300, pCAF and GCN5 & Tocris Bioscience & 4903 & $5 \mu \mathrm{M}$ \\
\hline
\end{tabular}

SAHA, suberoylanilide hydroxamic acid; TSA, trichostatin A.

Antibodies. Antibodies against the following proteins were used for FACS, immunoblotting and IF staining: MICA/B (6D4; BioLegend), mouse IgG2a isotype Ctrl (MOPC-173), Annexin V (BioLegend), pChk1 S345, Chk1, Ku80 (all from Cell Signaling Technology, Inc., Beverly, MA, USA) and E2F1 (C-20; Santa Cruz Biotechnology, Inc., Santa Cruz, CA, USA).

NK-mediated cytotoxicity assay. The NK-92 cell line which was used as an effector was obtained from the American Type Culture Collection (ATCC; Manassas, VA, USA) and maintained in Alpha-MEM (Gibco) containing $12.5 \%$ (v/v) horse serum, $12.5 \%(\mathrm{v} / \mathrm{v})$ fetal bovine serum and human recombinant IL-2. The cytotoxicity of NK-92 against T98G cells was assessed using the DELFIA EuTDA Cytotoxicity Assay Reagent kit AD0116 (PerkinElmer, Inc., Waltham, MA, USA). The experiment was performed following the manufacturer's instructions. Briefly, NK-92 cells used as effector cells were cultured with anti-CD314 (1D11) or mouse IgG1 (MOPC-21) (both from BioLegend) for $30 \mathrm{~min}$ before mixing the target cells. T98G cells used as target cells were cultured with BATDA for $30 \mathrm{~min}$ at RT, then the cells were washed 3 times with PBS containing 2\% newborn calf serum. BATDA-labeled T98G cells were plated onto round-bottom 96 well plates at a density of 5,000 cells/well and mixed with effector cells at various effector/target ratios. Spontaneous release was assessed in $\mathrm{T} 98 \mathrm{G}$ cells incubated with culture medium only, and maximum release was measured in T98G cells incubated for $30 \mathrm{~min}$ with $2 \%$ Triton X-100. After incubation for $4 \mathrm{~h}, 20 \mathrm{ml}$ of supernatant of each well were harvested for assessment of released TDA. The time-resolved fluorescence was assessed by EnVision (PerkinElmer, Inc.). The percentage of cytotoxicity was calculated by (Experimental release - Spontaneous release)/(Maximum release - Spontaneous release) x 100 .

Cell proliferation assay. Cell viability following vorinostat treatment was determined using Cell Proliferation kit I (Roche, Mannheim, Germany).

Statistical analysis. Statistical significance in the FACS, cell proliferation and NK-mediated cytotoxicity assays were determined using Student's two-tailed t-test or the Mann-Whitney U test, both in SigmaPlot 12.0.

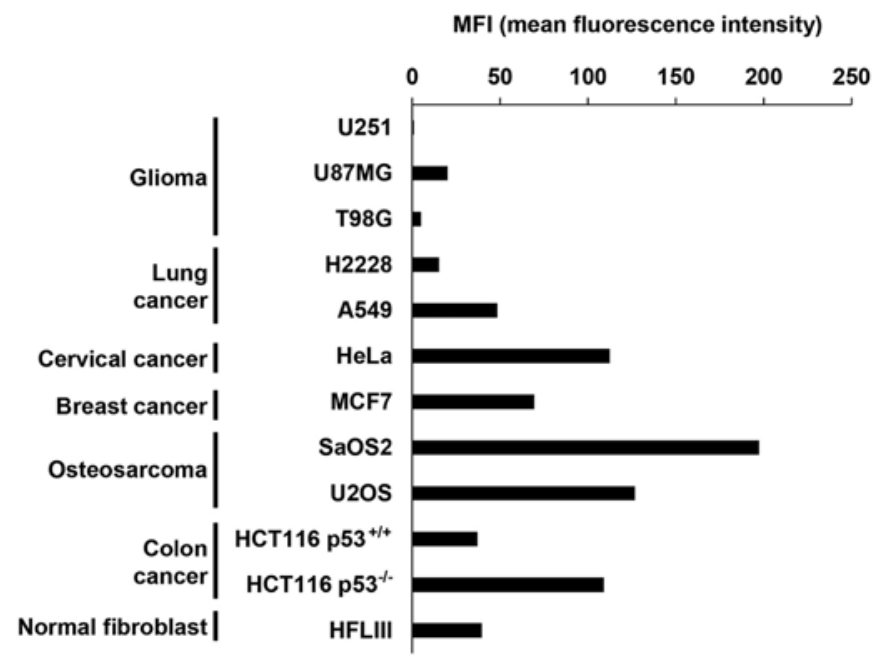

Figure 1. Variable expression of MICA/B expression in cancer cells with no DNA damage. Expression of MICA/B on the surface of glioma, lung, cervical and breast cancer, osteosarcoma and colon cancer cells, and on the surface of normal primary fibroblast cells without DNA damage was examined using flow cytometry. Data are representative of $>2$ independent experiments, all with similar results.

\section{Results}

Cancer cell lines exhibit a wide range of DNA damageinduced $M I C A / B$ expression, independent of tissue type and $R b / p 53$ status. Cell surface expression of MICA/B is upregulated after DNA damage (7). Previous studies have reported that spontaneous MICA/B expression varies widely among cancer cell lines (19-21). In the present study, to investigate variety of DNA damage-induced MICA/B expression among cancer cells, in relation to tissue type and tumor-suppressor gene status, MICA/B surface expression was examined in several cancer cell lines following exposure to IR. To assess human MICA/B expression, levels of MICA/B on the cell surface were analyzed by FACS. To ascertain that we were analyzing MICA/B expression in living cells, we assessed only PI and Annexin V double-negative cells (data not shown). Consistent with previous studies, the basal level of MICA/B expression in the absence of DNA damage varied among the cell types (Fig. 1). At $24 \mathrm{~h}$ after $10 \mathrm{~Gy}$ X-rays, we found that H2228, U2OS, U87MG, HeLa and SaOS2 cells exhibited high levels of MICA/B induction, whereas lower 

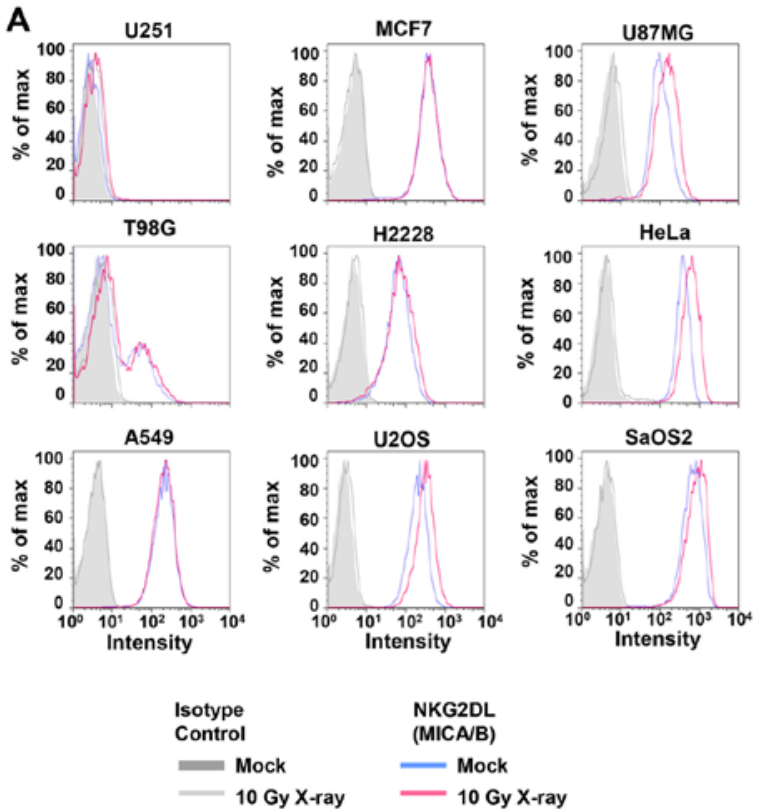

B

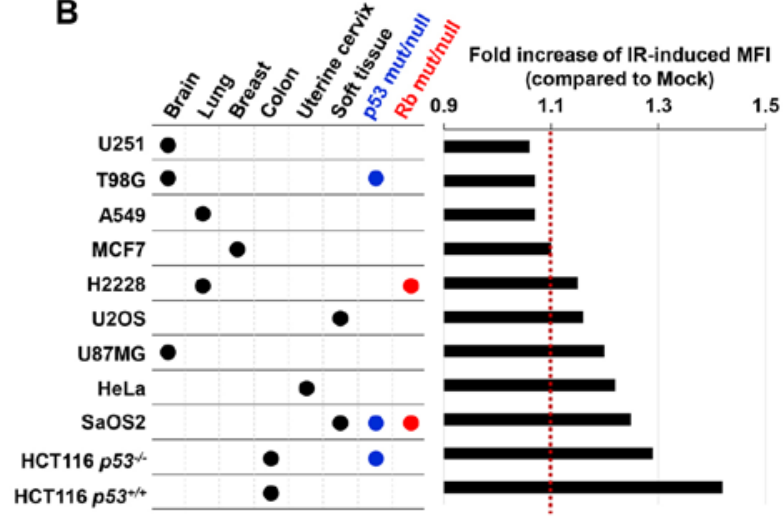

C
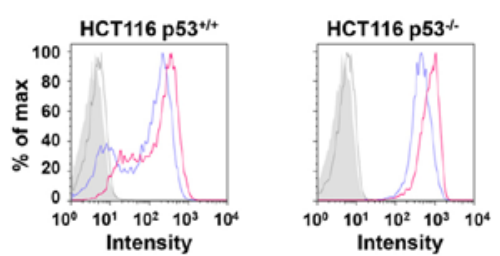

Figure 2. Cancer cell lines exhibit a wide range of variation in DNA damage-induced MICA/B expression. (A) Surface expression of MICA/B was examined in 9 cancer cell lines. Cells were analyzed using FACS following MICA/B staining, $24 \mathrm{~h}$ after 10 Gy X-irradiation. (B) IR-induced MFI (mean fluorescence intensity) was quantitated using FlowJo. The IR-induced fold increase in MFI was calculated by dividing the MFI in irradiated cells by the MFI in non-irradiated cells. In the present study, cell lines with ratios $<1.1$ or $>1.1$ were categorized as IR-insensitive or -sensitive, respectively. (C) IR-induced MICA/B is p53-independent. MICA/B surface expression in HCT116 wt or p53-knockout cells was analyzed by FACS 24 h after 10 Gy X-irradiation. Similar results were obtained in $>2$ independent experiments in all panels.

levels of induction were observed in U251, T98G, A549 and MCF7 cells (Fig. 2A and B). Notably, X-ray-induced MICA/B expression was not obviously correlated with tissue types (Fig. 2A and B). We also investigated MICA/B expression in relation to the status of tumor-suppressor genes, $\mathrm{Rb}$ and $\mathrm{p} 53$. However, we did not observe any correlation between mutation status and MICA/B expression (Fig. 2B and C).

X-ray-induced DSBs are mostly repaired by either HR or non-homologous end joining (NHEJ) within a few hours after X-rays (10). Therefore, we wondered whether DNA damage signaling following $\mathrm{X}$-irradiation was insufficient to induce MICA/B in insensitive cancer cells since the DNA damage is rapidly repaired, and the signal is consequently not maintained for a long period of time $(17,22)$. To address this issue, we tested whether heavy ion irradiation could activate MICA/B in insensitive T98G or A549 cell lines. In contrast to X-rays, heavy ion irradiation induces complex DNA lesions that delay the speed of DSB repair $(23,24)$. However, the insensitive cell lines did not express MICA/B even after carbon-ion irradiation (Fig. 3A). High levels of DNA damage signaling after carbon-ion irradiation were confirmed by Chk1 S345 phosphorylation (pChk1), since Chk1 has a central role in the upregulation of MICA/B expression after DNA damage (Fig. 3B) (7). The ATM/ATR dependency of this effect was confirmed by the decrease in pChk1 in the presence of an ATM- or ATR-specific inhibitor (Fig. 3C) (25). To further confirm the poor responsiveness in insensitive cells, we treated insensitive T98G cells with aphidicolin, a DNA polymerase inhibitor, which induces DNA damage by blocking DNA replication (26). Similarly to IR, aphidicolin treatment did not activate MICA/B in insensitive T98G cells (Fig. 3D and E). These data reveal that the limited response of MICA/B expression in insensitive cell lines is due to neither IR-induced damage complexity nor the type of DNA damage. Instead, other factors are likely involved in the suppression of DNA damage-dependent MICA/B activation.

Inhibition of histone deacetylase activity restores DNA damage-induced $M I C A / B$ expression in insensitive cancer cells. Next, we speculated that the insensitivity may be dependent on the suppression of the MICA/B gene expression due to an alteration in chromatin structure in cancer cells (27-29). To test this idea, we conducted a screening for compounds that restored MICA/B expression by targeting chromatin remodeling factors in insensitive T98G cells (Fig. 4A and Table I). The results revealed that vorinostat, an HDACi, substantially restored IR-dependent MICA/B induction in insensitive T98G (Fig. 4A and B) and U251 cells (data not shown), although the drug exerted little effect on other insensitive A549 and MCF7 cell lines (data not shown). In contrast, in sensitive U2OS and SaOS2 cells, IR also increased MICA/B expression even further in the presence of vorinostat (Fig. 4C). Consistent with previous findings, vorinostat alone increased MICA/B expression in insensitive as well as sensitive cells (Fig. 4 and Table II) (30-32). Similarly to vorinostat, trichostatin A (TSA; HDAC inhibitor) and the histone methyltransferase inhibitors; chaetocin (Suv39 inhibitor) and Bix-01294 (G9a inhibitor), which also relieve chromatin compaction, increased MICA/B expression on their own, and more importantly, treatment with these inhibitors restored IR-dependent MICA/B induction in insensitive cells (Fig. 4A and B, and Tables I and II). By contrast, neither DNA methyltransferase nor histone acetyltransferase (HAT) inhibitors affected MICA/B expression (Fig. 4A and B, and 


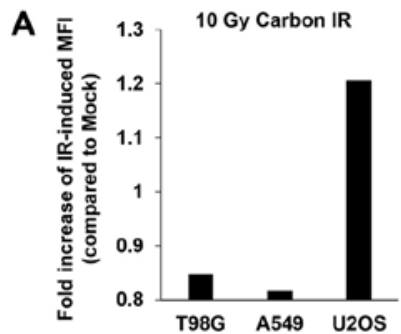

D

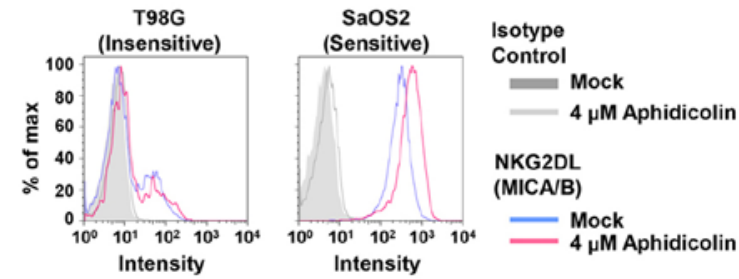

B

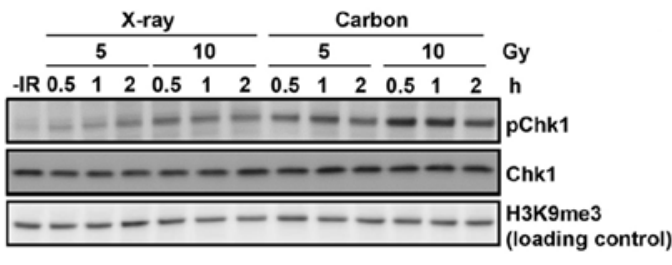

C

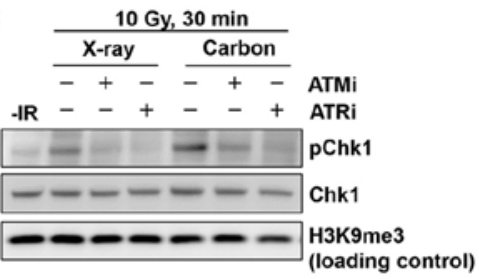

E

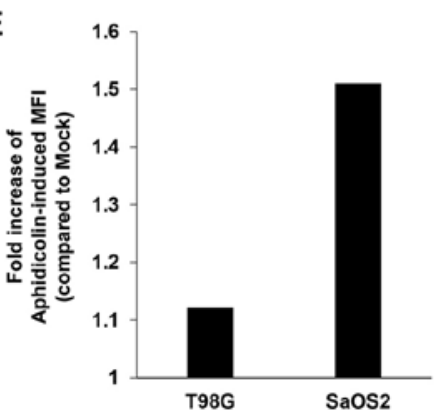

Figure 3. Limited responsiveness of MICA/B expression is observed in insensitive cell lines independent of the type of DNA damage. (A) Carbon IR, which produces complex DNA damage, induced MICA/B expression in U2OS (sensitive), but not in T98G and A549 (insensitive) cells. MICA/B expression was analyzed by FACS $24 \mathrm{~h}$ after 10 Gy carbon IR. (B) Carbon IR effectively induces DNA damage signaling. Phosphorylation of Chk1 S345 in A549 cells was examined by immunoblotting. (C) Phosphorylation of Chk1 S345 in A549 cells is ATM/ATR-dependent after both X-rays and carbon IR. (D) A similar response to MICA/B expression in T98G (insensitive) and SaOS2 (sensitive) cells was observed after treatment with aphidicolin, a DNA polymerase inhibitor, which induces DNA replication damage in the S phase. (E) Fold increase in aphidicolin-induced MFI from the experiment described in D. Similar results were obtained in $>2$ independent experiments in all panels.

A
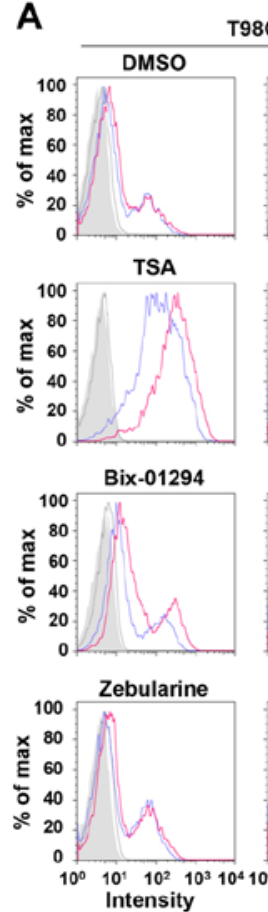

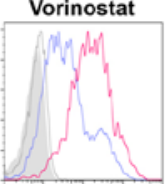

Chaetocin

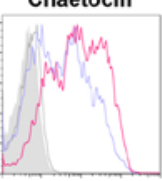

5-Aza-cytidine

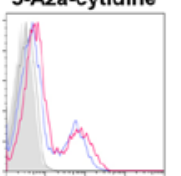

NU9056

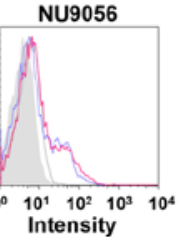

B Isotype
Control Mock
$10 \mathrm{~Gy}$ NKG2DL Mock $10 \mathrm{~Gy}$
X-ray
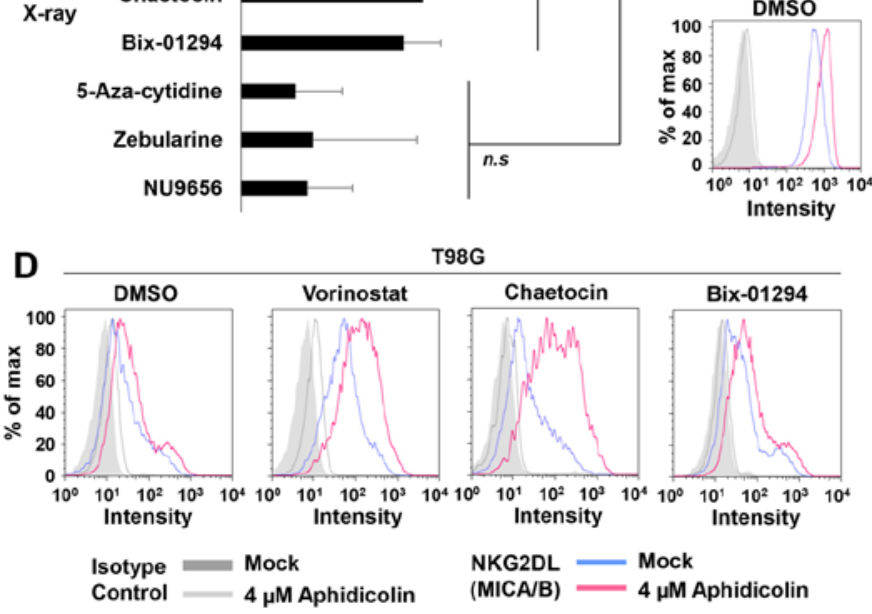

C
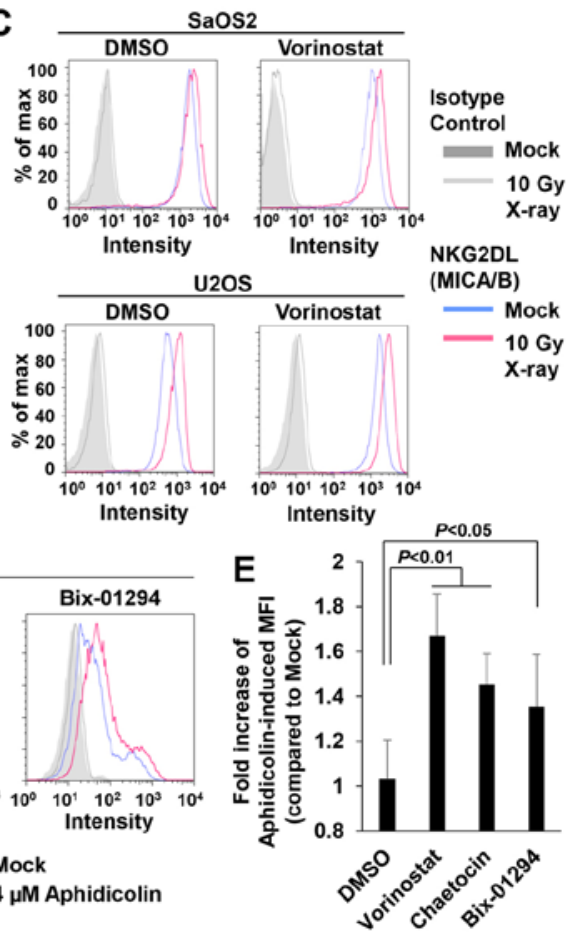

Figure 4. Screening analysis reveals that inhibition of the HDAC/Suv39/G9a pathway restores MICA/B expression in insensitive cancer cells after DNA damage. (A) MICA/B surface expression in insensitive T98G cells was analyzed by FACS at $24 \mathrm{~h}$ after irradiation with $10 \mathrm{~Gy}$. Vorinostat ( $1 \mu \mathrm{M}$ ), TSA $(100 \mathrm{nM})$, chaetocin $(50 \mathrm{nM})$, Bix-01294 $(5 \mu \mathrm{M}), 5$-aza-cytidine $(1 \mu \mathrm{M})$, zebularine $(10 \mu \mathrm{M})$, decitabine $(10 \mu \mathrm{M})$ or NU9056 $(10 \mu \mathrm{M})$ was added $2 \mathrm{~h}$ before irradiation with $10 \mathrm{~Gy}$ X-rays. Prior to analysis, cells were incubated for $24 \mathrm{~h}$ in the presence of the drugs. (B) Fold increase in IR-induced MFI from the experiment described in A. (C) MICA/B expression in sensitive SaOS 2 and U2OS cells was analyzed by FACS $24 \mathrm{~h}$ after 10 Gy irradiation $+/-1 \mu \mathrm{M}$ vorinostat. (D) MICA/B expression in insensitive T98G cells was analyzed $24 \mathrm{~h}$ after the addition of $4 \mu \mathrm{M}$ aphidicolin $+/-1 \mu \mathrm{M}$ vorinostat, $5 \mu \mathrm{M}$ Bix- 01294 or $50 \mathrm{nM}$ chaetocin. (E) Fold increase in aphidicolin-induced MFI from the experiment described in D. Similar results were obtained in $>2$ independent experiments in all panels.

Tables I and II). Although the effects of these inhibitors may be influenced by the conditions of the drug treatment, e.g., concentration or duration, our screening analysis revealed that inhibition of HDAC/Suv39/G9a was able to restore 
Table II. MFI in T98G, U2OS and SaOS2 cells in the presence of inhibitors +/- X-ray.

\begin{tabular}{llrrc}
\hline Cell line & Reagent & $\begin{array}{r}\text { MFI } \\
(-\mathrm{IR})\end{array}$ & $\begin{array}{c}\text { MFI } \\
(10 \mathrm{~Gy})\end{array}$ & $\begin{array}{c}\text { Fold increase of } \\
\text { IR-induced } \\
\text { MFI }\end{array}$ \\
\hline T98G & DMSO & 3.65 & 3.79 & 1.02 \\
& Vorinostat & 18.94 & 32.13 & 1.76 \\
& TSA & 67.66 & 108.0 & 1.60 \\
& Chaetocin & 8.59 & 13.13 & 1.44 \\
& Bix-01294 & 4.37 & 5.72 & 1.38 \\
& 5-Aza-cytidine & 4.76 & 4.54 & 0.99 \\
& Zebularine & 5.48 & 5.94 & 1.06 \\
& NU9056 & 4.92 & 4.95 & 1.03 \\
U2OS & DMSO & 84.70 & 105.50 & 1.25 \\
& Vorinostat & 190.89 & 251.0 & 1.31 \\
& DMSO & 168.91 & 207.61 & 1.23 \\
& Vorinostat & 389.26 & 493.79 & 1.27 \\
\hline
\end{tabular}

MFI, mean fluorescence intensity; IR, ionizing radiation; DMSO, dimethyl sulfoxide; TSA, trichostatin A.

and enhance DNA damage-induced MICA/B expression. To further consolidate the notion that HDAC/Suv39/G9a inhibition restores MICA/B expression in insensitive cells, we analyzed the MICA/B surface expression +/- HDAC, Suv39 or G9a inhibitor after aphidicolin treatment (Fig. 4D and E). Similar to the result after IR, aphidicolin-induced MICA/B expression was restored by HDAC/Suv39/G9a inhibition.

Restoration of MICA/B expression by HDACi is dependent on ATR and E2F1, a transcription factor that responds to DNA damage. DNA damage-dependent NKG2DL activation requires ATM/ATR (7). Therefore, we investigated whether the restoration of MICA/B expression by HDAC inhibition is dependent on these kinases. As expected, treatment with the ATR inhibitor (ATRi) decreased IR-dependent MICA/B expression restored by HDACi (Fig. 5A and B). In mice, NKG2DL expression is controlled by the transcription factor $\mathrm{E} 2 \mathrm{~F}$ (33). To assess the involvement of E2F1 in the restoration of IR-induced MICA/B expression by vorinostat, we evaluated
MICA/B expression following depletion of E2F1 by siRNA. Depletion of E2F1 in insensitive cells decreased IR-dependent MICA/B expression in the presence of HDACi (Fig. 5C-E). Thus, our results indicate that restoration of MICA/B expression by vorinostat is mediated by ATR-dependent DNA damage signaling, and that transcription is regulated by the E2F1 pathway.

Low-dose HDACi restores DNA damage-dependent MICA/B expression. MICA/B expression in cancer cells is increased following treatment with HDACi alone (30-32). Inhibition of HDAC alters global chromatin structure, resulting in dysregulation of gene expression (34). Such dynamic genome alterations sometimes cause severe side-effects in normal cells. Therefore, high concentrations of HDACi should be avoided in order to decrease side-effects in normal tissues (35). One advantage of radiotherapy is that it can directly target tumors; consequently, identification of the minimal dose of HDACi that can restore/enhance MICA/B expression following IR may facilitate clinical applications (36). Thus, we titrated vorinostat against insensitive cells to determine the lowest concentration of HDACi that can restore IR-induced MICA/B expression. We selected vorinostat for the titration analysis since treatment with TSA, chaetocin and Bix-01294 caused severe cell toxicity compared with vorinostat (data not shown). In addition, vorinostat has been used in clinical practice (37). Similar to the data in Fig. 4A and B, vorinostat alone increased MICA/B expression in insensitive cells in a dose-dependent manner (Table III). Notably, the titration analysis revealed that $0.5-2 \mu \mathrm{M}$ vorinostat restored DNA damage-dependent MICA/B expression in insensitive T98G cells (Fig. 6A and Table III). IR-dependent enhancement was not observed in the presence of $10 \mu \mathrm{M}$ vorinostat, possibly due to saturation of transcriptional activity (Fig. 6A and Table III). In contrast, $1 \mu \mathrm{M}$ vorinostat did not induce MICA/B expression in normal human fibroblast cells after IR (Fig. 6B). Next, we investigated whether treatment with the lower concentration of vorinostat causes toxicity in cancer cells or normal fibroblast cells. A concentration of $10 \mu \mathrm{M}$ vorinostat markedly curtailed cell growth in both cancer and normal cells (Fig. 6C and D). Although 1-2 $\mu \mathrm{M}$ vorinostat decreased the growth rate of normal fibroblast cells by $\sim 50 \%$, we confirmed that the growth inhibition was significantly lower than that induced by the $10-\mu \mathrm{M}$ vorinostat treatment (Fig. 6D).

Table III. MFI in T98G cells using vorinostat.

\begin{tabular}{lccccc}
\hline Cell line & Reagent & Dose $(\mu \mathrm{M})$ & MFI (-IR) & MFI (10 Gy) & $\begin{array}{c}\text { Fold increase of } \\
\text { IR-induced MFI }\end{array}$ \\
\hline T98G & Vorinostat & 0 & 3.88 & 4.38 & 1.13 \\
& & 0.5 & 7.76 & 9.61 & 1.24 \\
& 1.0 & 10.56 & 13.63 & 1.29 \\
& 2.0 & 19.93 & 32.45 & 1.63 \\
& 10.0 & 50.45 & 61.39 & 1.22 \\
\hline
\end{tabular}

MFI, mean fluorescence intensity; IR, ionizing radiation. 
A
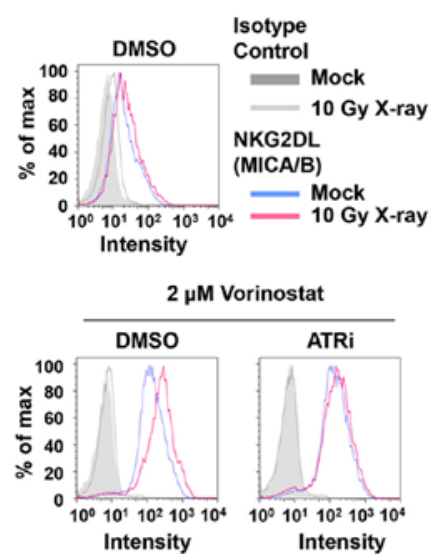

B

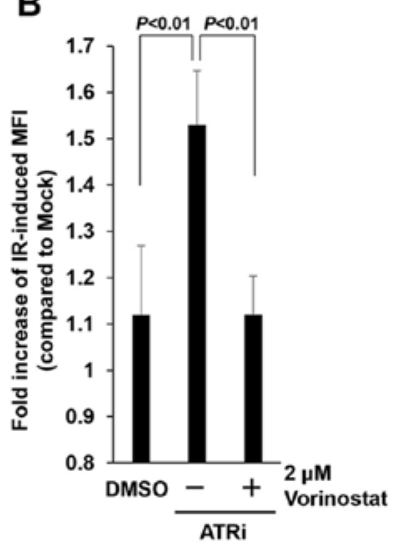

C

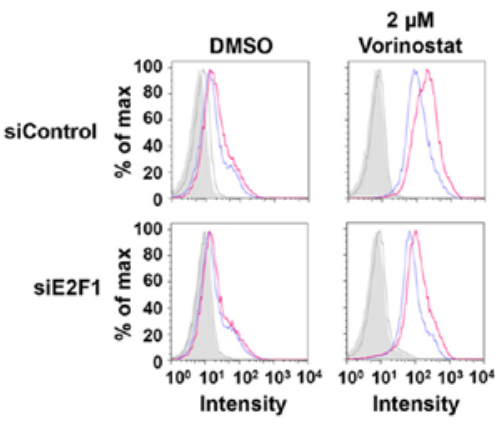

E
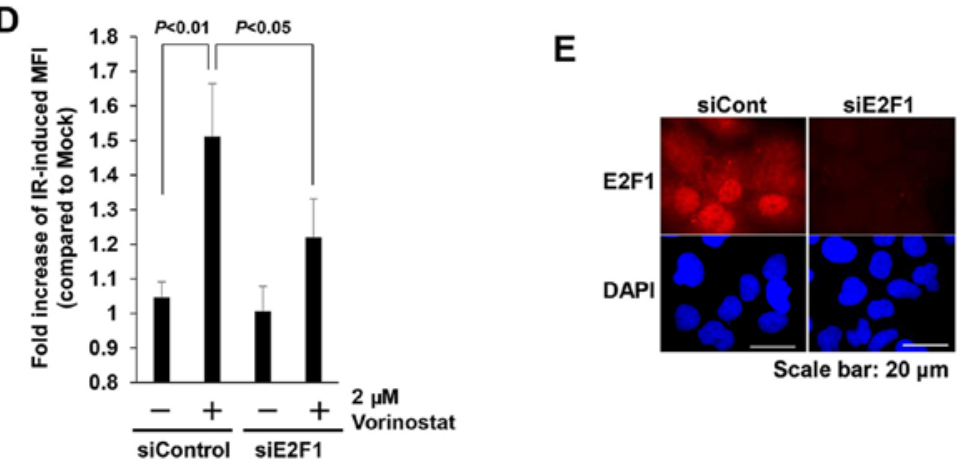

Figure 5. Restoration of IR-induced MICA/B expression in insensitive cells is dependent on ATR and E2F1. (A and B) MICA/B surface expression in insensitive T98G cells was analyzed by FACS $24 \mathrm{~h}$ after $10 \mathrm{~Gy}$ irradiation $+/$ - dimethyl sulfoxide (DMSO) or $10 \mu \mathrm{M} \mathrm{ATRi}$, all in the presence of $2 \mu \mathrm{M}$ vorinostat. (C) Insensitive T98G cells subjected to E2F1 siRNA were irradiated with $10 \mathrm{~Gy}+/-2 \mu \mathrm{M}$ vorinostat. (D) Fold increase in IR-induced MFI from the experiment described in C. (E) Knockdown efficiency of E2F1 was confirmed by immunofluorescence staining. Similar results were obtained in $>2$ independent experiments in all panels.

A
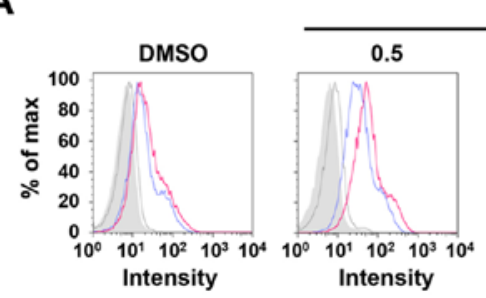

T98G + Vorinostat $(\mu \mathrm{M})$
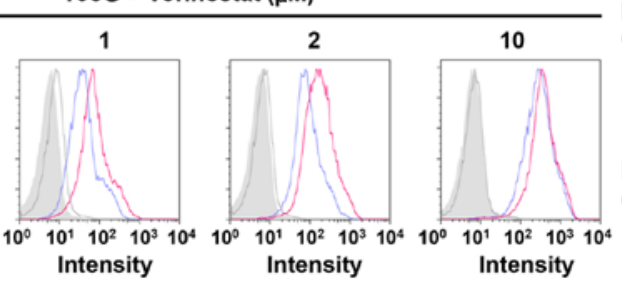

Isotype

Mock

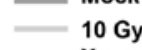

X-ray

B
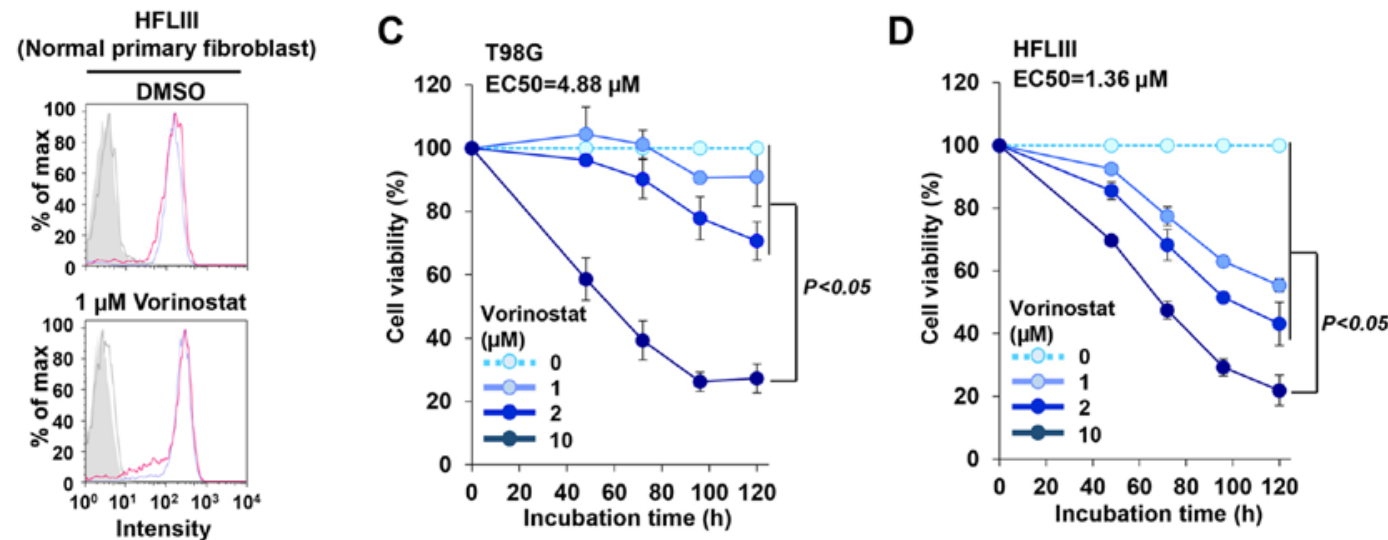

Figure 6. A dose of vorinostat that is non-lethal in normal primary fibroblast cells is sufficient to restore IR-induced MICA/B surface expression. (A) Titration of vorinostat to determine the dose required for restoration of MICA/B expression was performed in insensitive T98G cells after irradiation with $10 \mathrm{~Gy}$ X-rays (B) MICA/B expression in HFLIII (normal primary fibroblast) cells was analyzed in the presence of $1 \mu \mathrm{M}$ vorinostat at $24 \mathrm{~h}$ after $10 \mathrm{~Gy} \mathrm{X}$-rays. (C and D) The cell viability of T98G and HFLIII cells, respectively, was analyzed in the presence of vorinostat. Cells were incubated with 1,2 or $10 \mu \mathrm{M}$ vorinostat for the indicated times. Similar results were obtained in $>2$ independent experiments in all panels. 
A

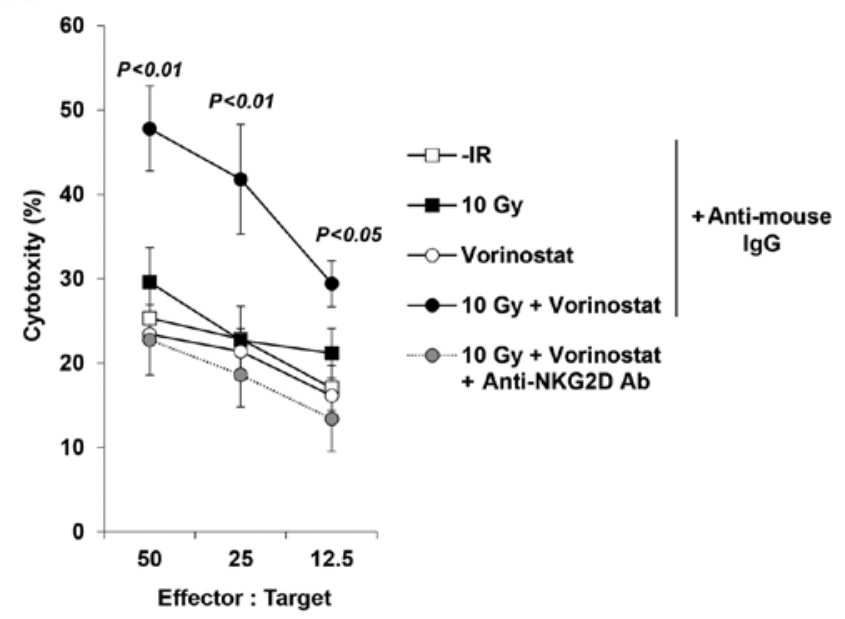

B

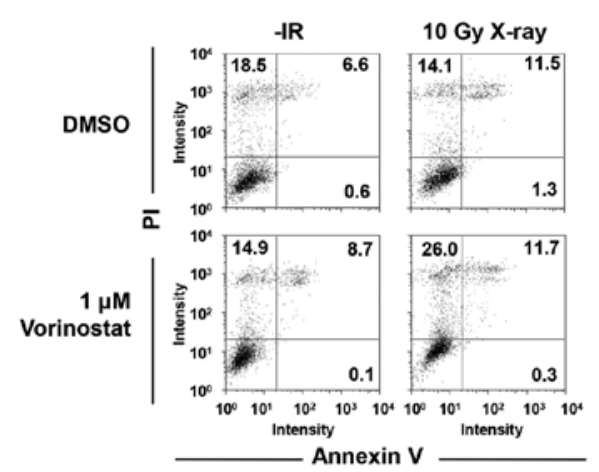

Figure 7. Histone deacetylase inhibitor (HDACi) restores IR-induced NK-mediated cytotoxicity in insensitive cells. (A) The cytotoxicity of NK-92 cells against T98G cells. The horizontal axis indicates the ratio of the number of effector cells vs. the number of target cells. (B) Cell viability of T98G cells post-treatment with IR or vorinostat. The $\mathrm{x}$-axis shows the intensity of Annexin V staining, whereas the y-axis shows the intensity of PI staining (as detected by flow cytometry). Results are representative of $>2$ independent experiments, all with similar results.

$H D A C i$ restores $I R$-induced NK-mediated cytotoxicity in insensitive cells. Next, to confirm whether the restored MICA/B expression affects NK cell-mediated cytotoxicity, an NK cell cytotoxicity assay was performed with/without vorinostat in insensitive T98G cells. Treatment with vorinostat substantially restored the activity of T98G lysis, suggesting the restoration of T98G cell susceptibility to NK-92-mediated killing (Fig. 7A). Notably, the increase in cytotoxicity was critically dependent on the NKG2D-MICA/B interaction, since it was completely abolished by addition of a blocking anti-NKG2D Ab (Fig. 7A), whereas addition of an isotype control $\mathrm{mAb}$ did not significantly affect NK-92-mediated lysis (Fig. 7A). We examined levels of PI and Annexin V to confirm that $10 \mathrm{~Gy}+/-1 \mu \mathrm{M}$ vorinostat in the absence of NK-92 cells did not cause cell death at $24 \mathrm{~h}$ after IR, suggesting that neither IR nor HDACi (or both) affected cell viability in this time range of analysis (Fig. 7B).

Collectively, these data strongly demonstrate that DNA damage-dependent MICA/B expression and NK cell cytotoxicity in insensitive cancer cells can be restored by inhibition of the HDAC pathway.

\section{Discussion}

NKG2DL is constitutively expressed in various tumor cells $(19,21)$. In addition, ATM/ATR signaling contributes to NKG2DL expression induced by DNA-damaging agents both in vivo and in vitro (7). In the present study, we investigated whether cancer cell lines showed distinct responsiveness of MICA/B expression after DNA damage. Our data provide the first demonstration that there is considerable variation in MICA/B expression among cancer cells in response to DNA damage. Notably, neither the complexity of IR-induced damage nor the type of DNA damage influenced the restoration of DNA damage-dependent MICA/B expression in insensitive cancer cells. This observation supports the important notion that stimulation by DNA damage alone cannot effectively overcome the suppressive phenotype in insensitive cancer cells.
Our drug screening analysis demonstrated that histone $\mathrm{H} 3 \mathrm{~K} 9$ modification is a key process involved in the restoration and enhancement of MICA/B expression, even in the absence of DNA damage. HDACs deacetylate multiple lysine residues of histones, resulting in chromatin compaction (38). Therefore, inhibition of HDAC activity leads to chromatin relaxation. HDAC inhibition also affects the responsiveness of gene expression. Since gene silencing is caused by the chromatin condensation in the promoter region, forced genome-wide relaxation by HDACi treatment can restore gene expression even when the DNA at the promoter region is highly methylated $(28,39)$. Similar to the role of HDAC, Suv39/G9a, a methyltransferase of histone $\mathrm{H} 3 \mathrm{~K} 9$, promotes chromatin compaction. HDACs and Suv39/G9a function in the same axis, and the balance of their activities controls chromatin structure. In the drug screening analysis, we found that inhibition of Suv39/G9a activity also enhanced MICA/B expression. In the present study, we revealed that inhibition of HDAC, Suv39 or G9a activity increased MICA/B expression in both insensitive and sensitive cell lines in the absence of DNA damage (30-32). Recently, Baragaño Raneros et al demonstrated that MICA/B is highly methylated in several acute myeloid leukemia cell lines (28). From these observations, we proposed the idea that MICA/B is frequently downregulated by gene silencing as a consequence of tumor development, however, by the inhibition of HDAC/Suv39/G9a activity, MICA/B gene expression may be restored by the reactivation of MICA/B transcription at the relaxed promoter region.

In the present study, we found that low-dose HDACi sufficiently restored DNA damage-dependent MICA/B expression, which was dependent on DNA damage signaling via the ATR pathway. ATR activates Chk1, which transduces downstream signals to control gene expression in response to DNA damage. In addition, we found that IR-induced MICA/B expression requires E2F1. Collectively, these data reveal that the ATR/Chk1 signal promotes E2F1-dependent transcriptional activity, which is required for MICA/B expression. However, future studies may be required to determine the 
mechanism of the signaling cascade from ATR/Chk1 to E2F1. Notably, DNA damage alone did not induce MICA/B expression in insensitive cells; rather, it was restored in the presence of HDACi. Moreover, we wondered whether the restoration of MICA/B expression is dependent on IR or HDACi when it is combined. Although the current data may not fully answer the question, we believe that the results suggest that the MICA/B gene reacquires IR-responsiveness when MICA/B gene silencing is restored by vorinostat. In addition, we found that the surface expression of MICA/B following DNA damage was not restored by HDAC inhibition in H2228, A549 or MCF7 cell lines (data not shown). These data may suggest that the non-responsiveness of MICA/B expression in these cell lines is caused by other mechanisms, e.g., mutations in the MICA/B gene or dysfunction of the MICA/B protein; this issue may be addressed in a future study. Previous studies demonstrated that HDAC inhibition prevents DSB repair (40). Therefore, we assessed whether inhibition of NHEJ affects the expression of MICA/B in cancer cells, however, we did not see an obvious change in expression (data not shown). Further studies are required to assess precisely how DNA repair influences MICA/B expression following DNA damage.

Vorinostat and panobinostat are approved in the US for the treatment of cutaneous $\mathrm{T}$ cell lymphoma and multiple myeloma, respectively. Thus, single-agent administration of an HDACi has already been shown as useful cancer treatment (39). Conversely, the biggest advantage of radiotherapy is the ability to target the tumor without systemic side-effects. However, despite the improvement of therapeutic efficacy due to development of novel radiotherapy technologies, metastases can occur, since it is technically unfeasible to irradiate all the disseminated micro-metastatic cancer cells. It is therefore important to find the best cohort for radiotherapy. The therapeutic benefit of radiotherapy in combination with HDACi has already been shown in vivo and in vitro $(41,42)$. Our findings support the idea that HDACi may be a good cohort for radiotherapy, since the combination therapy may treat not only a gross tumor, but also micro-metastatic cancer cells by immune response. Alternatively, upregulation of MICA/B can be a productive marker for the efficacy of therapeutic agents. For instance, by combining HDACi treatment with radioimmunoconjugate therapy, another potential application in preclinical and clinical settings (43), the exposed ligands may be used as a target for elimination of cancer cells.

In summary, this is the first study revealing the wide variation in MICA/B surface expression in cancer cell lines following DNA damage. Notably, inhibition of HDAC activity was able to restore DNA damage-dependent MICA/B expression in insensitive cells. Our analysis revealed that the restored MICA/B expression was mediated by ATR and E2F1 signaling. Previous studies revealed that HDACi treatment exerts its anticancer effects via multiple mechanisms, including prevention of DNA repair and promotion of the adaptive immune response $(44,45)$. In addition to these effects, our data demonstrate that HDACs may contribute to MICA/B (NKG2D)-mediated antitumor effects after IR. Thus, combination therapy using HDACi and radiotherapy or DNA damage-inducing chemotherapy represent valid and feasible approaches to cancer therapy.

\section{Acknowledgements}

We would like to thank Michie Akaishizawa, Yoshimi Omi and Shiho Nakanishi for their technical and administrative assistance in the present study. The present study was supported by the Astellas Foundation for Research on Metabolic Disorders, the Terumo Life Science Foundation, and the JSPS KAKENHI (grant no. 26701005). The present study was carried out as part of the Research Project with Heavy Ions at NIRS-HIMAC and GHMC. We would like to thank the NIRS-HIMAC and GHMC engineering staff for providing support for our heavy ion experiments.

\section{References}

1. Gras Navarro A, Björklund AT and Chekenya M: Therapeutic potential and challenges of natural killer cells in treatment of solid tumors. Front Immunol 6: 202, 2015.

2. Robert C, Long GV, Brady B, Dutriaux C, Maio M, Mortier L, Hassel JC, Rutkowski P, McNeil C, Kalinka-Warzocha E, et al: Nivolumab in previously untreated melanoma without $B R A F$ mutation. N Engl J Med 372: 320-330, 2015.

3. Brahmer J, Reckamp KL, Baas P, Crinò L, Eberhardt WE, Poddubskaya E, Antonia S, Pluzanski A, Vokes EE, Holgado E, et al: Nivolumab versus docetaxel in advanced squamous-cell non-small-cell lung cancer. N Engl J Med 373: 123-135, 2015.

4. Bauer S, Groh V, Wu J, Steinle A, Phillips JH, Lanier LL and Spies T: Activation of NK cells and T cells by NKG2D, a receptor for stress-inducible MICA. Science 285: 727-729, 1999.

5. Raulet DH and Guerra N: Oncogenic stress sensed by the immune system: Role of natural killer cell receptors. Nat Rev Immunol 9: 568-580, 2009.

6. Lanier LL: NKG2D receptor and its ligands in host defense. Cancer Immunol Res 3: 575-582, 2015.

7. Gasser S, Orsulic S, Brown EJ and Raulet DH: The DNA damage pathway regulates innate immune system ligands of the NKG2D receptor. Nature 436: 1186-1190, 2005.

8. Fine JH, Chen P, Mesci A, Allan DS, Gasser S, Raulet DH and Carlyle JR: Chemotherapy-induced genotoxic stress promotes sensitivity to natural killer cell cytotoxicity by enabling missingself recognition. Cancer Res 70: 7102-7113, 2010.

9. Soriani A, Zingoni A, Cerboni C, Iannitto ML, Ricciardi MR, Di Gialleonardo V, Cippitelli M, Fionda C, Petrucci MT, Guarini A, et al: ATM-ATR-dependent up-regulation of DNAM-1 and NKG2D ligands on multiple myeloma cells by therapeutic agents results in enhanced NK-cell susceptibility and is associated with a senescent phenotype. Blood 113: 3503-3511, 2009.

10. Shibata A and Jeggo PA: DNA double-strand break repair in a cellular context. Clin Oncol 26: 243-249, 2014.

11. Shiloh Y: ATM and related protein kinases: Safeguarding genome integrity. Nat Rev Cancer 3: 155-168, 2003.

12. Rhind N: Changing of the guard: How ATM hands off DNA double-strand break signaling to ATR. Mol Cell 33: 672-674, 2009.

13. Shinkai Y and Tachibana M: H3K9 methyltransferase G9a and the related molecule GLP. Genes Dev 25: 781-788, 2011.

14. Melcher M, Schmid M, Aagaard L, Selenko P, Laible G and Jenuwein T: Structure-function analysis of SUV39H1 reveals a dominant role in heterochromatin organization, chromosome segregation, and mitotic progression. Mol Cell Biol 20: 3728-3741, 2000.

15. Tachibana M, Sugimoto K, Fukushima T and Shinkai Y: Set domain-containing protein, G9a, is a novel lysine-preferring mammalian histone methyltransferase with hyperactivity and specific selectivity to lysines 9 and 27 of histone H3. J Biol Chem 276: 25309-25317, 2001.

16. Clayton A, Mitchell JP, Court J, Linnane S, Mason MD and Tabi Z: Human tumor-derived exosomes down-modulate NKG2D expression. J Immunol 180: 7249-7258, 2008.

17. Shibata A, Moiani D, Arvai AS, Perry J, Harding SM, Genois MM, Maity R, van Rossum-Fikkert S, Kertokalio A, Romoli F, et al: DNA double-strand break repair pathway choice is directed by distinct MRE11 nuclease activities. Mol Cell 53: 7-18, 2014. 
18. Nakajima NI, Hagiwara Y, Oike T, Okayasu R, Murakami T, Nakano $\mathrm{T}$ and Shibata A: Pre-exposure to ionizing radiation stimulates DNA double strand break end resection, promoting the use of homologous recombination repair. PLoS One 10 : $\mathrm{e} 0122582,2015$.

19. Groh V, Rhinehart R, Secrist H, Bauer S, Grabstein KH and Spies T: Broad tumor-associated expression and recognition by tumor-derived gamma delta T cells of MICA and MICB. Proc Natl Acad Sci USA 96: 6879-6884, 1999.

20. Diefenbach A and Raulet DH: Strategies for target cell recognition by natural killer cells. Immunol Rev 181: 170-184, 2001.

21. Pende D, Rivera P, Marcenaro S, Chang CC, Biassoni R, Conte R, Kubin M, Cosman D, Ferrone S, Moretta L, et al: Major histocompatibility complex class I-related chain A and UL16-binding protein expression on tumor cell lines of different histotypes: Analysis of tumor susceptibility to NKG2D-dependent natural killer cell cytotoxicity. Cancer Res 62: 6178-6186, 2002.

22. Deckbar D, Jeggo PA and Löbrich M: Understanding the limitations of radiation-induced cell cycle checkpoints. Crit Rev Biochem Mol Biol 46: 271-283, 2011.

23. Shibata A, Conrad S, Birraux J, Geuting V, Barton O, Ismail A Kakarougkas A, Meek K, Taucher-Scholz G, Löbrich M, et al: Factors determining DNA double-strand break repair pathway choice in G2 phase. EMBO J 30: 1079-1092, 2011.

24. Schmid TE, Dollinger G, Beisker W, Hable V, Greubel C, Auer S, Mittag A, Tarnok A, Friedl AA, Molls M, et al: Differences in the kinetics of gamma-H2AX fluorescence decay after exposure to low and high LET radiation. Int J Radiat Biol 86: 682-691, 2010.

25. Jazayeri A, Falck J, Lukas C, Bartek J, Smith GC, Lukas J and Jackson SP: ATM- and cell cycle-dependent regulation of ATR in response to DNA double-strand breaks. Nat Cell Biol 8: 37-45, 2006.

26. Rothkamm K, Krüger I, Thompson LH and Löbrich M: Pathways of DNA double-strand break repair during the mammalian cell cycle. Mol Cell Biol 23: 5706-5715, 2003.

27. Kato N, Tanaka J, Sugita J, Toubai T, Miura Y, Ibata M, Syono Y, Ota S, Kondo T, Asaka M, et al: Regulation of the expression of MHC class I-related chain A, B (MICA, MICB) via chromatin remodeling and its impact on the susceptibility of leukemic cells to the cytotoxicity of NKG2D-expressing cells. Leukemia 21 2103-2108, 2007.

28. Baragaño Raneros A, Martín-Palanco V, Fernandez AF, Rodriguez RM,Fraga MF,Lopez-Larrea C and Suarez-Alvarez B Methylation of NKG2D ligands contributes to immune system evasion in acute myeloid leukemia. Genes Immun 16: 71-82, 2015.

29. Baylin SB: DNA methylation and gene silencing in cancer. Nat Clin Pract Oncol 2 (Suppl 1): S4-S11, 2005.

30. Skov S, Pedersen MT, Andresen L, Straten PT, Woetmann A and Odum N: Cancer cells become susceptible to natural killer cell killing after exposure to histone deacetylase inhibitors due to glycogen synthase kinase-3-dependent expression of MHC class I-related chain A and B. Cancer Res 65: 11136-11145, 2005.

31. Armeanu S, Bitzer M, Lauer UM, Venturelli S, Pathil A, Krusch M, Kaiser S, Jobst J, Smirnow I, Wagner A, et al: Natural killer cell-mediated lysis of hepatoma cells via specific induction of NKG2D ligands by the histone deacetylase inhibitor sodium valproate. Cancer Res 65: 6321-6329, 2005.
32. Diermayr S, Himmelreich H, Durovic B, Mathys-Schneeberger A, Siegler U, Langenkamp U, Hofsteenge J, Gratwohl A, Tichelli A, Paluszewska M, et al: NKG2D ligand expression in AML increases in response to HDAC inhibitor valproic acid and contributes to allorecognition by NK-cell lines with single KIR-HLA class I specificities. Blood 111: 1428-1436, 2008.

33. Jung H, Hsiung B, Pestal K, Procyk E and Raulet DH: RAE-1 ligands for the NKG2D receptor are regulated by E2F transcription factors, which control cell cycle entry. J Exp Med 209: 2409-2422, 2012 .

34. Dokmanovic M, Clarke C and Marks PA: Histone deacetylase inhibitors: Overview and perspectives. Mol Cancer Res 5: 981-989, 2007.

35. Su H, Altucci L and You Q: Competitive or noncompetitive, that's the question: Research toward histone deacetylase inhibitors. Mol Cancer Ther 7: 1007-1012, 2008.

36. Avallone A, Piccirillo MC, Delrio P, Pecori B, Di Gennaro E, Aloj L, Tatangelo F, D'Angelo V, Granata C, Cavalcanti E, et al: Phase $1 / 2$ study of valproic acid and short-course radiotherapy plus capecitabine as preoperative treatment in low-moderate risk rectal cancer-V-shoRT-R3 (Valproic acid - short Radiotherapy - rectum 3rd trial). BMC Cancer 14: 875, 2014.

37. Krug LM, Kindler HL, Calvert H, Manegold C, Tsao AS, Fennell D, Öhman R, Plummer R, Eberhardt WE, Fukuoka K, et al: Vorinostat in patients with advanced malignant pleural mesothelioma who have progressed on previous chemotherapy (VANTAGE-014): A phase 3, double-blind, randomised, placebo-controlled trial. Lancet Oncol 16: 447-456, 2015.

38. Gaszner M and Felsenfeld G: Insulators: Exploiting transcriptional and epigenetic mechanisms. Nat Rev Genet 7: 703-713, 2006.

39. West AC and Johnstone RW: New and emerging HDAC inhibitors for cancer treatment. J Clin Invest 124: 30-39, 2014

40. Miller KM, Tjeertes JV, Coates J, Legube G, Polo SE, Britton S and Jackson SP: Human HDAC1 and HDAC2 function in the DNA-damage response to promote DNA nonhomologous endjoining. Nat Struct Mol Biol 17: 1144-1151, 2010.

41. Blattmann C, Oertel S, Thiemann M, Dittmar A, Roth E, Kulozik AE, Ehemann V, Weichert W, Huber PE, Stenzinger A, et al: Histone deacetylase inhibition sensitizes osteosarcoma to heavy ion radiotherapy. Radiat Oncol 10: 146, 2015.

42. Kim JH, Shin JH and Kim IH: Susceptibility and radiosensitization of human glioblastoma cells to trichostatin A, a histone deacetylase inhibitor. Int J Radiat Oncol Biol Phys 59: 1174-1180, 2004.

43. Cornelissen B, Kersemans V, Darbar S, Thompson J, Shah K, Sleeth K, Hill MA and Vallis KA: Imaging DNA damage in vivo using gammaH2AX-targeted immunoconjugates. Cancer Res 71: 4539-4549, 2011

44. Munshi A, Kurland JF, Nishikawa T, Tanaka T, Hobbs ML, Tucker SL, Ismail S, Stevens C and Meyn RE: Histone deacetylase inhibitors radiosensitize human melanoma cells by suppressing DNA repair activity. Clin Cancer Res 11: 4912-4922, 2005.

45. West AC, Smyth MJ and Johnstone RW: The anticancer effects of HDAC inhibitors require the immune system. Oncoimmunology 3: e27414, 2014 